\title{
ESTADÍSTICAS LABORALES Y LA INCORPORACIÓN DE TICS EN LA UNIVERSIDAD NACIONAL DE ROSARIO, ARGENTINA
}

\author{
Nora Lac Prugent \\ Rosario State University, Argentina \\ nlacprug@fcecon.unr.edu.ar
}

Este trabajo muestra la aplicación de herramientas estadisticas en el análisis de fuentes de datos oficiales para el estudio de la relación trabajo-condiciones de vida. De esta manera, es fruto de la experiencia en la enseñanza de la Estadística aplicada a las Ciencias Sociales plasmado en el dictado de la signatura Metodologías Cuantitativas Aplicadas en la Universidad Nacional de Rosario. Así, resulta de interés que aquellos que están cursando un postgrado aborden el tratamiento integral de temas de candente actualidad como los relacionados con las condiciones socio- económico por la que están atravesando los habitantes de la región. Adicionalmente, surge la necesidad de relacionar la enseñanza tradicional de la Estadística con las nuevas herramientas computacionales. Se posibilita, de esta manera, no sólo preparar a los maestrandos para el ejercicio profesional, sino también ampliar y flexibilizar el razonamiento propio de esta ciencia.

\section{JUSTIFICACIÓN Y FUNDAMENTACIÓN}

La motivación de esta ponencia responde a la intención de mejorar progresivamente la enseñanza de la Estadística, en especial en carreras en las cuales cumple un rol instrumental. Con tal fin se presentan dos problemas motivadores con la intención de introducir la lógica estadísticacomputacional como herramienta de análisis en las Ciencias Sociales. La modalidad de enseñanza planteada es posible únicamente si la metodología de trabajo en las clases se basa en la activa participación de los alumnos. El presente escrito se plantea, entonces, respondiendo a tal metodología.

Cabe indicar que las encuestas sobre condiciones de vida procuraron constituirse en un insumo significativo para el análisis socio-económico en la Argentina y ser una herramienta de consulta obligada para una gran diversidad de investigadores, técnicos y funcionarios del área social.

En la actualidad, en cualquier campo de aplicación, se necesitan habilidades para manejar el gran volumen de información disponible, en las diferentes áreas de trabajo, frente a mercados globalizados, ya no son suficientes la intuición o la práctica para delinear políticas exitosas. Las decisiones deben estar basadas en información objetiva, relevada y tratada con métodos científicos válidos

La metodología didáctica propuesta proporciona a los participantes, herramientas estadístico- metodológicas para el tratamiento estadístico de información proveniente del muestreo por encuestas y de estudios observacionales; así como, herramientas para el análisis y la evaluación de resultados, en especial en la gestión pública. De este modo, intenta dar respuesta a la pregunta: ¿Por qué es necesario estudiar métodos cuantitativos?

\section{OBJETIVOS Y METODOLOGÍA}

Esta asignatura de posgrado dictada bajo la forma de taller tiene como finalidad compartir con los docentes de la Universidad Nacional de Rosario, el análisis sobre la problemática del abordaje de la Estadística Aplicada en el ámbito académico universitario, permanente y continua preocupación tanto desde el campo de la investigación como de la capacitación docente.

Este curso se centra en el análisis de propuestas pedagógicas basadas en trabajos que tienen dicha orientación a fin de que los docentes puedan repensar las prácticas del uso de los métodos cuantitativos en los que participan, como problemática de su ámbito de acción. La intención es que comiencen a elaborar estrategias didácticas que contemplen nuevos abordajes para la interpretación estadística de la problemática que los circunda. 
Objetivos Generales del Curso

- Conocer la terminología y lenguaje estadístico para una efectiva comunicación con el especialista y manejar las técnicas de la Estadística para la correcta interpretación de resultados y rápida acción sobre el problema.

- Desarrollar la capacidad de análisis sobre la situación socioeconómica de los habitantes de una región o país.

- Conocer y usar las herramientas estadísticas para la presentación de datos y acciones en el área social.

- Manejar documentos teórico-metodológicos de la colecta de datos por encuesta (muestra, diccionario de variables: definición nominal y definición operacional, codificación, manual de entrenamiento del encuestador), bases de datos y análisis de datos con procesadores estadísticos. Uso de fuentes de datos estadísticos oficiales.

- Interpretar los resultados de pruebas de inferencia estadística y tomar decisiones con incertidumbre acotada

- Capacitar para la evaluación de información y resultados obtenidos a partir de una encuesta.

\section{Objetivos Especificos}

Se espera que el alumno:

- reconozca las distintas herramientas que brindan la estadística descriptiva y la inferencial,

- adquiera conocimientos que le permitan análisis estadísticos descriptivos tanto en artículos académicos como en informes de gestión,

- reconozca los alcances y los límites del estudio cuantitativo y la validez de los resultados de los mismos,

- identifique el tipo de datos y seleccione las medidas de estadística descriptiva apropiadas para sintetizar información proveniente de diferentes fuentes y expresada en diferentes escalas de medición,

- defina la población objeto de una investigación por muestreo y las variables de interés a considerar en la construcción de los instrumentos de colecta de información,

- categorice a la encuesta como un medio científico para el conocimiento de la realidad,

- logre conciencia sobre la importancia del diseño de la encuesta como un proceso que responde a objetivos fijados,

- forme criterio para la elección de la encuesta como metodología y para la inserción del investigador en el proceso de su diseño e implementación.

Los contenidos estadísticos abordados abarcan los siguientes conceptos: el papel de los métodos cuantitativos en el proceso de investigación; fuentes y bases de datos: los instrumentos para la colecta de información y la estructura de una base de datos; tipos de variables; codificación de respuestas; exploración de las bases, problemas de consistencia; el tratamiento de la falta de respuesta. Finalizando con los métodos descriptivos: exploración y organización de los datos e interpretación de los datos con los correspondientes resúmenes estadísticos.

De este modo, este curso integra:

- la adquisición de conocimientos básicos en estadística descriptiva, con una introducción a la estadística inferencial y

- la aplicación de esos conocimientos en el análisis de datos sociales por encuesta, mediante el adiestramiento en el programa estadístico para ciencias sociales: Statistical Package for Social Sciences (SPSS).

Es evidente que el programa es algo ambicioso. En este contexto y gracias a la posibilidad de utilizar la herramienta computacional, se decidió la incorporación de la práctica en computadoras para, por un lado, paliar el factor olvido de los alumnos y poder así avanzar en las aplicaciones socio-económicas. De este modo, la incorporación de las tecnologías de la información agiliza el desarrollo más completo relacionado con la aplicación requerida.

De esta manera, se trabaja en forma interactiva con la computadora, centrando el aprendizaje en la actividad del alumno. El docente es un facilitador del aprendizaje, a cargo de consultas actuando sólo cuando los comandos de ayuda del sistema sean insuficientes. 


\section{LAS GUÍAS DE TRABAJO PROPUESTAS}

Esta exposición muestra la aplicación de herramientas estadísticas en el análisis de fuentes de datos oficiales para el estudio de la relación trabajo-condiciones de vida. Concretamente, incursiona en el abordaje del análisis cuantitativo de la Encuesta Permanente de Hogares (EPH) en la República Argentina relevada por el Instituto Nacional de Estadística y Censos (INDEC).

Los enunciados que se relatan a continuación forman parte de los trabajos prácticos correspondientes a maestrandos cuya formación de grado proviene de psicología, ciencias de la educación, ciencias económicas, bioquímica, abogacía, antropología y estadística. Se han seleccionado porque permiten apreciar el valor de la herramienta computacional, en cuanto a rapidez de respuesta, en un proceso de construcción del conocimiento muy difícil dado lo interdisciplinario de la propuesta. El esquema metodológico está basado en la participación activa de los egresados usando el recurso de la discusión.

\section{Ejemplo 1: Familiarización con las bases de datos}

El ejercicio es individual. Consiste en seleccionar, de la base individuos de la EPH, un hogar familiar de cualquier tipo y describir las características de sus miembros. A fin de acotar el trabajo debe escoger un sólo módulo de los posibles: trabajo, educación, etc. La aplicación consta de dos partes.

- En la primera parte debe armar un texto descriptivo con todas las variables del módulo escogido. La búsqueda de la información se hará en clase con asistencia docente.

- En la segunda parte, debe reconocer para cada una de las variables el tipo y el nivel de medición que permite: categórica; numérica. Para esta parte pueden seleccionar las variables directamente desde el diccionario de variables, copiarlo en un procesador de texto y escribir al costado la identificación requerida.

\section{Ejemplo 2: Interpretación de los datos: resúmenes estadísticos}

Considere los siguientes dominios de estudio a partir de la base provista por el INDEC para el 4to. Trimestre 2014 del relevamiento correspondiente a la Encuesta Permanente de Hogares. En la primera parte del práctico se sugieren dos territorios, elija sólo uno de su preferencia; se apreciarán vivamente trabajos que reflejen originalidad en sus interpretaciones, recuerde que los trabajos deben realizarse en forma individual. Para cada uno de ellos presente tablas de frecuencias: absolutas, relativas, porcentuales y acumuladas; y gráficos acorde con el tipo de variable bajo estudio en cada ítem.

- Ocupados asalariados de 14 años y más. Región Pampeana / Aglomerado Gran Rosario

- Variables a considerar: tipo de establecimiento y monto por sueldos / jornales, salario familiar, horas extras, otras bonificaciones habituales y tickets, valores o similares percibidos en el mes de referencia.

- Para los datos numéricos utilizados consigne: el valor de la media aritmética, la mediana, el tercer decil, el primer cuartil, el tercer cuartil, el rango, el sexto decil, la mayor frecuencia absoluta y el valor de la frecuencia relativa acumulada al $70 \%$.

- Construya un diagrama de caja y bigotes, ¿cuáles son las cinco medidas resumen necesarias para construirlo? ¿Las distribuciones obtenidas son simétricas o asimétricas? Justifique.

\section{MODALIDAD DE DICTADO Y EVALUACIÓN}

El curso se desarrolla mediante clases teóricas-prácticas presenciales que combinan sesiones de visualización y análisis de los materiales de encuesta en pantalla y entrenamiento directo consistente en actividades de dos alumnos por computadora en base a prácticos grupales.

La evaluación se hace en base a los prácticos semi-presenciales los cuales integran conocimientos en los tres campos del curso: estadística, uso del software para el análisis de los materiales y datos de encuestas por muestreo. Los prácticos consisten en aplicaciones de los contenidos teóricos utilizando las bases de datos que aporta la cátedra. Los asistentes cuentan con el material de las encuestas y manuales de referencia del software para el entrenamiento en clase y la ejecución de los prácticos. 
El cupo de asistencia está en función de la disponibilidad del Laboratorio de Informática, cuyos asistentes pueden carecer de conocimientos previos de Estadística. La asistencia mínima requerida es del $75 \%$.

\section{EVALUACIÓN PRELIMINAR DE LA EXPERIENCIA}

Con el fin de evaluar el desarrollo de los cursos de postgrado se conformó un Comité de Evaluación. Después de recordar los objetivos de la reunión, los alumnos manifestaron diversas opiniones relativas al desarrollo de los mismos, al desempeño de los docentes, a las metodologías pedagógicas y al sistema de evaluación.

De sus respectivas evaluaciones individuales surge que aproximadamente las tres cuartas partes expresaron individualmente una evaluación muy positiva de su desarrollo.

Varios de los alumnos hicieron uso de la palabra, generalmente para hacer observaciones y efectuar propuestas a tomar en cuenta para la próxima cohorte. Las opiniones estuvieron asociadas directamente a su profesión: los comentarios más pertinentes provinieron de los graduados en ciencias sociales, puesto que todos ellos habían cursado, durante el grado, algunas de las materias que luego se desarrollaron más profundamente en la carrera de postgrado, y además cuentan con una sólida experiencia profesional en el área de la gestión.

A continuación se resumen algunos comentarios textuales recogidos durante la reunión relativa a la asignatura Metodologías Cuantitativas Aplicadas:

"Quienes lograron sortear las dificultades operativas, reconocieron la calidad académica de los docentes, consideraron que el curso les fue de utilidad y despertó su interés en manejar con mayor solvencia el software cada vez más utilizado en el medio académico".

Dado que los alumnos provienen de formación de grado muy diferentes: de las ciencias sociales y de las ciencias duras o con aplicaciones matemáticas, las dificultades del trabajo en laboratorio son leves y razonables para éstos últimos. También para ellos, el interés, la dinámica o el estímulo que produce el trabajo en laboratorio es altamente satisfactorio y la mayoría de los entrevistados manifiesta que la tarea resultó interesante. El resultado de la reunión, también indicó que: "no se deben dejar en un segundo plano los conceptos teóricos básicos acerca de los métodos cuantitativos, sobre los cuales muchos de los alumnos tenían pocos o insuficientes conocimientos". Efectivamente, la mayoría posee nulos o escasos conocimiento de Estadística.

Todos los asistentes priorizaron la importancia de la rapidez en el cálculo y la presentación sistematizada de grandes masas de datos. Manifestaron que una vez incorporada la teoría se pueden reafirmar y profundizar los conceptos. Por último, el alumno hace una muy buena valoración en cuanto a la utilidad de la herramienta computacional en el proceso del aprendizaje no sólo para esta asignatura sino en vista a futuras materias de la curricula.

En estos años se ha detectado en una etapa exploratoria, que los alumnos llegan al curso de postgrado con conocimientos previos de computación, pero sin el manejo de un software estadístico específico.

Además, los integrantes del Comité Evaluador indicaron que relativo a otras materias, las opiniones vertidas giraron sobre las siguientes acotaciones: "solicitaron que se considere la posibilidad de incluir profesionales con más experiencia en el tema y con una metodología pedagógica ajustada a un nivel de postgrado. Los alumnos manifestaron que el contenido fue muy limitado. Además las estrategias didácticas aplicadas por algunos docentes fueron cuestionadas debido a limitantes tales como lectura de power point o lectura de textos de leyes relevantes para el módulo, pero sin un proceso de análisis consistente y crítico".

En este estado de situación, ¿dónde se encuentra el equilibrio en cursos de postgrado procedentes de disciplinas tan heterogéneos? Hasta hoy, considero que prefiero una dura crítica relativa al nivel elevado del cursado y a la importancia operativa asignada al software que recibir las apreciaciones sobre la búsqueda de profesores para nivel de postgrado.

\section{CONCLUSIONES}

Se insiste en puntualizar que la práctica con la computadora es el complemento de los desarrollos teóricos realizados en el aula. Hay que recordar que esta ejercitación está dirigida a usar la computadora como una "calculadora inteligente" y esto por sí sólo no es suficiente para realizar 
el aprendizaje de un curso de Metodologías Cuantitativas Aplicadas, debiéndose integrar los conocimientos brindados con una adecuada bibliografía y buen desarrollo teórico.

Esta integración de conocimientos ha permitido satisfacer los objetivos iniciales. Esto es, por medio de la herramienta computacional, incrementar la utilización de la Estadística motivando a los estudiantes de postgrado a través de un enfoque actualizado, a la resolución sus problemas específicos.

Desde el lugar de la enseñanza, se puede argumentar que la herramienta computacional constituye un verdadero aliado del profesor como facilitador del proceso pedagógico; aún con el riesgo de asignar una gran importancia operativa al software, desconocido para la mayoría de los alumnos.

Es así como la sinergia entre los contenidos (qué queremos que aprendan los estudiantes) y la tecnología (equipamiento informático utilizado) contribuye a mejorar la pedagogía (cómo ayudar a aprender), permitiendo a los alumnos una capacitación acorde a los requerimientos de la sociedad actual.

\section{REFERENCIAS}

Agresti, A., \& Finlay, B. (1997). Statistical Methods for the Social Sciences. New Jersey: Prentice Hall

Berenson, M., Levine D., \& Krehbiel, T. (2001). Estadística para administración. $2^{\mathrm{a}}$ Edición Prentice Hall.

Cleveland, W. (1993). Visualizing Data. New Jersey, Hobart Press

Esquivel, V. La "Economía del cuidado": un recorrido conceptual. [en línea]. Buenos Aires: Universidad Nacional de General Sarmiento, s/f, [citado el 30 de abril 2011], s/f, p.13. http://www.cnm.gov.ar

Faur, E. "Género, masculinidades y políticas de conciliación familia-trabajo". Nómadas [en línea]. Colombia: Universidad Central, $\mathrm{n}^{\circ}$ 24, [citado 2 de mayo 2011], abril 2006, p. 130-141. http://redalyc.uamex.mx/src/inicio/ArtPdfRed.jsp?!Cve $=105116598012$

Levin, R., \& Rubin, D. (2004) Estadística para Administración y Economía. México, Pearson Education, $7^{\mathrm{a}}$ Edición.

Instituto Nacional de Estadística y Censos (INDEC). Acerca del método utilizado para la medición de la pobreza en Argentina. Documento preparado por la Dirección Nacional de Encuestas de Hogares.Metodología $N^{\circ} 13$ - República Argentina. Ministerio de Economía. Secretaría de Política Económica.Encuesta Permanente de Hogares - Mercado de trabajo, principales indicadores $h t t p: / / w w w . i n d e c . m e c o n . g o v . a r$

Lac Prugent, N., \& Añaños, M. C. (2012). Estrategias Metodológicas para los Estudios Regionales y del Mercado de Trabajo. Ponencia Estudios Regionales y Mercados de Trabajo, SIMEL, Santa Fe.

Lac Prugent, N., \& Añaños, M. C. (2011). Fuentes de datos secundarios para el análisis de la relación trabajo-condiciones de vida. Ponencia $10^{\circ}$ Congreso Nacional de Estudios del Trabajo, ASET, Buenos Aires.

PNUD. Objetivos del Milenio. Rendición de cuentas. Buenos Aires: PNUD [citado el 30 de marzo 2011], agosto 2010. Disponible en Internet: $h t t p: / / w w w . u n d p . o r g . a r / d o c s / O D M 2010 . p d f$

Presidencia de la Nación (2003). Objetivos de Desarrollo del Milenio. Argentina. La oportunidad para su reencuentro

Rowntree, D. (1984) Introducción a la estadística. Un enfoque no matemático. Ed. Norma.

Sen, Amartya K. (1992), "Sobre conceptos y medidas de pobreza", en revista Comercio Exterior, $\mathrm{n}^{\circ}$ 4, pp. 310-322, México

United Nations Statistics Division. Guide to produce Statistics on Time Use: Mesuring Paid and Unpaid Work (2005). New York: UNSTATS [citado el 1 de febrero 2010] http://unstats.un.org/unsd/cr/registry/regcst.asp? $C I=231 \& L g$

Webster, A. (2000) Estadística aplicada a los negocios y la economía. Bogotá: McGraw-Hill Interamericana S.A.

Zeisel, H. (1962) Dígalo con números. México: Fondo de Cultura Económica. 